\title{
Perceptual Learning Increases the Strength of the Earliest Signals in Visual Cortex
}

\author{
Min Bao (鲍敏), ${ }^{1}$ Lin Yang, ${ }^{2}$ Cristina Rios, ${ }^{2}$ Bin He, ${ }^{2}$ and Stephen A. Engel ${ }^{1}$ \\ Departments of ${ }^{1}$ Psychology and ${ }^{2}$ Biomedical Engineering, University of Minnesota, Minneapolis, Minnesota 55455
}

Training improves performance on most visual tasks. Such perceptual learning can modify how information is read out from, and represented in, later visual areas, but effects on early visual cortex are controversial. In particular, it remains unknown whether learning can reshape neural response properties in early visual areas independent from feedback arising in later cortical areas. Here, we tested whether learning can modify feedforward signals in early visual cortex as measured by the human electroencephalogram. Fourteen subjects were trained for $>24 \mathrm{~d}$ to detect a diagonal grating pattern in one quadrant of the visual field. Training improved performance, reducing the contrast needed for reliable detection, and also reliably increased the amplitude of the earliest component of the visual evoked potential, the $\mathrm{C} 1$. Control orientations and locations showed smaller effects of training. Because the $\mathrm{C} 1$ arises rapidly and has a source in early visual cortex, our results suggest that learning can increase early visual area response through local receptive field changes without feedback from later areas.

\section{Introduction}

Learning in most perceptual tasks has a component specific to the trained stimulus. For example, training in orientation discrimination transfers only partially to stimuli that differ in orientation or visual field location from the trained ones (e.g., Karni and Sagi, 1991; Fahle, 2004). This specificity led to the hypothesis that some component of perceptual learning depends upon local changes in the receptive fields of neurons in early visual cortex (e.g., Karni and Sagi, 1991; Poggio et al., 1992).

The hypothesis remains controversial, however, having received only mixed support from neurophysiology studies using animals. While relatively large effects of learning have been found using single-unit recording in a later sensorimotor area (Law and Gold, 2008) and moderate effects have been found in intermediate visual area V4 (Yang and Maunsell, 2004; Raiguel et al., 2006), effects in primary visual cortex (V1) have been mixed, reported in different studies as absent (Ghose et al., 2002), small (Schoups et al., 2001), large (Hua et al., 2010), or dependent upon feedback from higher cortical areas (Li et al., 2004). Neuroimaging studies in humans have found larger effects of learning on V1 activity (Schwartz et al., 2002; Furmanski et al., 2004; Kourtzi et al., 2005; Sigman et al., 2005; Mukai et al., 2007; Yotsumoto et al., 2008), but these could be due to feedback from later areas, possibly depending upon selective attention, form recognition, or other relatively high-level processes. Some psychophysical results also suggest that perceptual learning may primarily affect higher levels of processing (e.g., Xiao et al., 2008; Lu et al., 2010).

\footnotetext{
Received Nov. 17, 2009; revised Sept. 8, 2010; accepted Sept. 13, 2010.

This work was supported in part by National Institutes of Health Grant R01 EB 007920. We thank Gordon E. Legge, Keith Jamison, and two anonymous reviewers for their helpful comments and Ken Paller and Satoru Suzuki for discussions on an earlier version of this project.

Correspondence should be addressed to Stephen A. Engel at the above address. E-mail: enge|@umn.edu.

D01:10.1523/JNEUROSCI.5703-09.2010

Copyright $\odot 2010$ the authors $\quad 0270-6474 / 10 / 3015080-05 \$ 15.00 / 0$
}

To test whether learning can produce local receptive field changes in early visual cortex, we measured the electroencephelogram (EEG), and focused on the earliest visual evoked potential (VEP) component, termed the C1 (Jeffreys and Axford, 1972). One important property of the $\mathrm{C} 1$ is that it reverses polarity depending on whether stimuli are presented to the upper or lower visual field. This is taken as evidence that $\mathrm{V} 1$ is its major generator (e.g., Martínez et al., 1999; Di Russo et al., 2002; Foxe and Simpson, 2002), though recently it has been suggested that $\mathrm{V} 2$ or $\mathrm{V} 3$ could also be the source (Ales et al., 2010). The $\mathrm{C} 1$ is also the earliest VEP component, taking $\sim 50-70 \mathrm{~ms}$ to rise above baseline after a visual stimulus. Because of these two properties, the $\mathrm{C} 1$ likely reflects "feedforward" response of neurons in early visual areas, which depends upon local processing of signals arriving into cortex (e.g., Gomez Gonzalez et al., 1994; Di Russo et al., 2003) (but see Foxe and Simpson, 2002).

We tested whether training a contrast detection task could modify the amplitude of the C1. Our results show that perceptual learning increased its amplitude, suggesting that training altered the locally determined receptive field properties of neurons in primary visual cortex.

\section{Materials and Methods}

\section{Participants}

Fourteen volunteers (five males, nine females) participated in the study. Volunteers received monetary compensation and gave informed consent. Procedures were approved by the University of Minnesota Office for the Protection of Research Subjects.

\section{Stimuli}

Stimuli were patches of sine-wave gratings whose edges were smoothed with a Gaussian filter. The patches subtended either 5 or 3.57 deg of visual angle and were centered either 7 or 5 deg away from the fixation (see below). All contained a spatial frequency of five cycles per stimulus. Stimuli for behavioral measurements were displayed on a CRT using 14 bit video (Cambridge Research Systems). Stimuli for ERP recordings were displayed on an LCD monitor with 8 bit video. Gamma curves were 
A
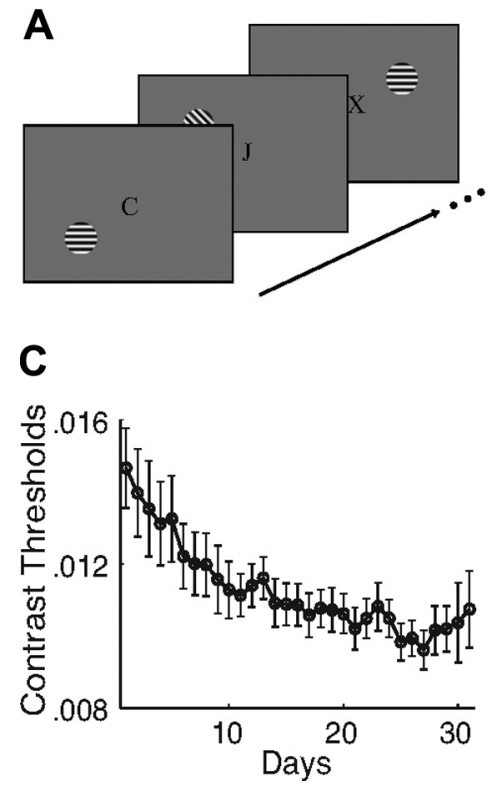

$\mathbf{B}$

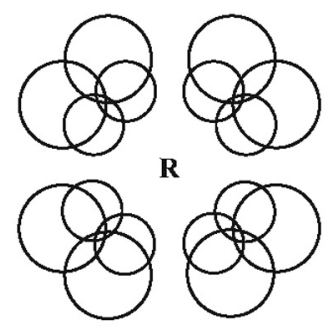

D

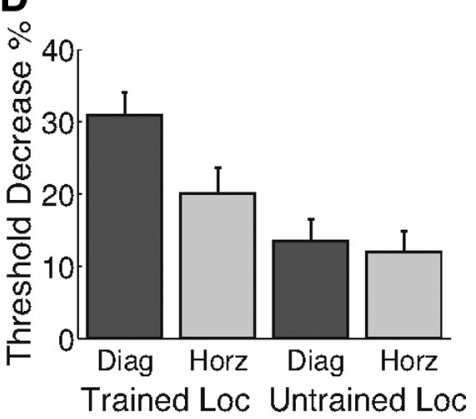

Figure 1. Methods and behavioral results. $\boldsymbol{A}$, Schematic of the RSVP task used during EEG recording. A central letter stream was presented, with letters changing at the rate of $4.17 \mathrm{~Hz}$. Subjects' task was to report the presence of an "X." Gratings were presented for 100 ms each, with a randomly varying stimulus onset asynchrony. The onsets of gratings and letters were asynchronous. Letters are not drawn to scale. $\boldsymbol{B}$, Circles indicate the 16 possible locations for stimulus presentation. One location in each quadrant was chosen for each subject, determined by a separate C1 mapping session. C, Behavioral training curve averaged across all subjects $(n=14)$. Error bars are \pm 1 SEM. $\boldsymbol{D}$, Percentage threshold decrease for each stimulus condition. Thresholds decreased most for the trained grating tested at the trained location.

measured for both displays (using a PhotoResearch PR-655), and were inverted in software.

\section{Procedure}

EEG. EEG data, digitized at $500 \mathrm{~Hz}$, were acquired from 64 scalp electrodes (Neuroscan) with an amplifier bandpass of DC to $70 \mathrm{~Hz}$ and a $60 \mathrm{~Hz}$ notch filter. Vertical electro-oculogram (VEO) was recorded by electrodes placed above and below the left eye. Horizontal electro-oculogram was recorded by electrodes placed at the left and right outer canthi, but only for six subjects. The reference electrode was placed on the top of the midline between electrodes CZ and CPZ. In all EEG sessions, a central rapid serial visual presentation (RSVP) task was used to control subjects' attention (Fig. 1A). Subjects pressed a button upon seeing an " $\mathrm{X}$ " in a central letter stream presented at $4.17 \mathrm{~Hz}$.

The $\mathrm{C} 1$ is difficult to observe in some individuals, perhaps due to the specific orientation and folding of their calcarine sulci. To overcome this variability, we probed for visual field locations that produced strong C1 responses in separate mapping sessions conducted before any training (Kelly et al., 2008). Subjects viewed checkerboard stimuli presented at 16 retinal locations along two circles (Fig. $1 B$ ). The visual field location in each quadrant that produced the most robust $\mathrm{C} 1$ response was selected for use in all later sessions.

In these subsequent sessions, full-contrast grating patches were presented at each selected location in two different orientations, 45 deg or horizontal, for a total of eight conditions. Patches were displayed one at a time for $100 \mathrm{~ms}$ with random phase. Patch condition was controlled using a nine-level $\mathrm{m}$-sequence (including a mean field condition). Patch presentations were separated by a randomly chosen interval of between 150 and $450 \mathrm{~ms}$. Stimuli were presented in runs that lasted $\sim 104 \mathrm{~s}$. Data from 32 runs were collected, yielding 1008 repetitions in each condition.

Psychophysics. We measured contrast detection thresholds using a two-interval forced-choice task. Each trial consisted of two $200 \mathrm{~ms}$ intervals separated by $200 \mathrm{~ms}$ and signaled by a tone. Subjects judged which interval contained a grating. Contrast was controlled by a staircase procedure. Six staircases were run for each of the eight conditions both before and after training, blocked in a random order that was counterbalanced across subjects.
Training. One quadrant was selected for training, counterbalanced across subjects. All subjects trained on the diagonally oriented stimulus. Training lasted for $>24 \mathrm{~d}$ (mean $28.64, \mathrm{SD}=2.44)$. Subjects performed the contrast detection task, as described above, once daily for 30-40 min (960 trials per day, mean of 27,497 total trials), viewing exclusively the diagonal stimulus at the chosen location.

\section{Analysis}

Behavior. To measure detection thresholds, Weibull functions were fit to the data pooled from all staircases in a given condition and session. Threshold was defined as the contrast at which the best-fitting function estimated performance to be $82 \%$ correct.

EEG. Data were analyzed using EEGLAB 6.01b (http://www.sccn.ucsd.edu/eeglab). Raw data were first filtered off-line with a bandpass filter that passed $0.1-30 \mathrm{~Hz}$. Data excursions exceeding $\pm 50 \mu \mathrm{V}$ at electrode VEO $(-100$ to $+300 \mathrm{~ms}$ ) were excluded from analysis, as were data surrounding the target letter " $X$ " in the RSVP task $(-100$ to +1200$)$ and surrounding a button press $(-600$ to +600$)$. Mean VEPs were computed for each stimulus orientation and location, by averaging from $100 \mathrm{~ms}$ before to $300 \mathrm{~ms}$ after stimulus onset.

The topography of $\mathrm{Cl}$ can vary between individuals, depending upon the geometry of the calcarine and placement of the electrode cap. But for almost every subject in every condition, a clear spatial peak in the topographic maps of voltage was visible within $60-90 \mathrm{~ms}$ after stimulus, near the scalp location where $\mathrm{C} 1$ is usually observed. In the few cases in which the time window did not include a clear peak, it was shifted by up to $30 \mathrm{~ms}$. To quantify $\mathrm{C} 1$ amplitude, we averaged the signals from the electrode with the largest peak amplitude in the time window, and the adjacent two electrodes with the strongest signals at the time of the peak (separately for each session; $71 \%$ of electrodes were identical in both sessions, for electrodes used, see supplemental Table S1, available at www. jneurosci.org as supplemental material). $\mathrm{C} 1$ amplitude was defined as the height of the peak in this average signal, and its latency was defined as its time to the peak.

To ensure that the changing electrode positions did not affect our results, we repeated our analyses using fixed electrode positions (supplemental Table S2, available at www.jneurosci.org as supplemental material). To pick these electrodes, we first noted for each session the electrode with the largest peak amplitude and the adjacent four electrodes with strongest peaks. We then identified all electrodes noted for both sessions, and selected for analysis the two with the strongest mean peak signals. This procedure did not change the overall pattern of results from that reported below.

For statistical analysis, amplitudes and latencies were averaged across the three untrained locations and compared to the trained location. At the trained location, all subjects showed clear peaks. At untrained locations, five subjects did not show clear peaks with $\mathrm{C} 1$ topography in the $30-120 \mathrm{~ms}$ time range in one of the untrained quadrants, and five subjects did not show $\mathrm{C} 1$ peaks in two untrained quadrants. Quadrants without $\mathrm{C} 1$ peaks were excluded from further analysis.

\section{Results}

Contrast detection thresholds and VEPs were measured before and after training for eight conditions comprising two stimulus orientations (trained diagonal and untrained horizontal) at four locations (trained visual field quadrant and three untrained quadrants).

\section{Behavior}

Training decreased detection thresholds, with the largest effects occurring for the trained orientation at the trained loca- 
tion. Figure $1 C$ shows average thresholds for the trained stimulus across training. Thresholds decreased by $\sim 30 \%$. Figure $1 D$ shows changes in threshold from the pretest to the posttest (for untrained locations, data were averaged across all three untrained quadrants). A component of learning was specific to the trained stimulus: Orientation (trained vs untrained) and location (trained vs untrained) showed a reliable interaction in an ANOVA conducted on the change scores $\left(F_{(1,13)}=18.36, p<\right.$ $0.001)$. Additionally, planned comparisons showed that thresholds decreased more for the trained orientation than for the control orientation at the trained location $\left.t_{(13)}=5.13, p<0.001\right)$ but not at untrained locations $\left(t_{(13)}=1.05, p>0.31\right)$. There was also evidence for a component of learning that was specific for location but generalized across orientation ( $t$ test for untrained orientation at trained location vs untrained locations, $t_{(13)}=2.79$, $p<0.015$ ).

Subjects performed the RSVP task with high accuracy during the EEG sessions [pretest: $96.0 \%$, mean reaction time $(\mathrm{RT})=568 \mathrm{~ms}$; posttest: $96.7 \%$, mean $\mathrm{RT}=564 \mathrm{~ms}]$. No significant difference was found when comparing performance $\left(t_{(13)}=1.03, p>0.321\right)$ and reaction time $\left.t_{(13)}=0.67, p>0.513\right)$ before and after training.

\section{VEP}

Early cortical responses showed a clear C1 component, which increased in amplitude following training. Figure 2 shows average topographies of the VEP for the trained stimulus before and after learning. These topographies show the polarity reversal as a function of stimulus visual field that is typical of the C1 (supplemental Fig. $\mathrm{S} 1$, available at www.jneurosci.org as supplemental material, shows similarly typical topographies for grand average VEPs computed across all conditions). The topographies also show clear effects of learning, visible as an increase in the height and width of the voltage peak.

The increase in $\mathrm{C} 1$ amplitude was specific for both orientation and location. Figure $3 A$ shows the time course of the average VEPs for trained and untrained orientations. $\mathrm{C} 1$ is the early peak that is negative for upper visual field stimuli and positive for lower visual field stimuli. The $\mathrm{C} 1$ for the trained orientation increased by a larger amount after training than did the untrained orientation.

Statistical analysis of the $\mathrm{C} 1$ amplitudes confirmed the specificity of learning. To compensate for different $\mathrm{C} 1$ magnitudes in upper and lower visual fields, amplitudes at each location were normalized by dividing by the amplitude of the untrained orientation in the pretest. Normalized amplitudes (Fig. $3 B, C$ ) were then entered into an ANOVA with factors orientation (trained, untrained), location (trained, untrained), and session (pretraining,
Upper left VF

Upper right VF
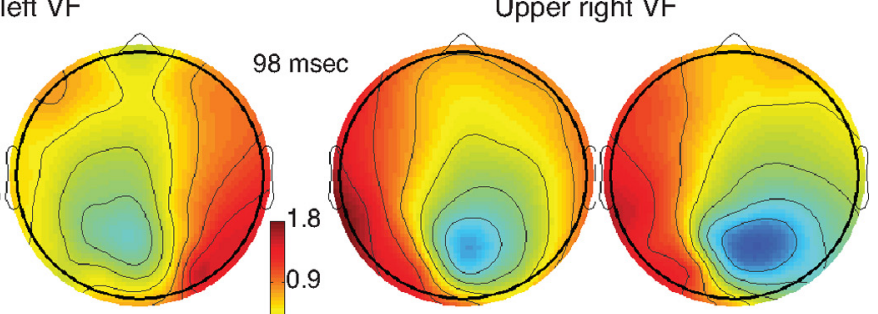

Pre

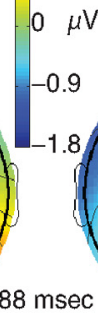

$88 \mathrm{msec}$

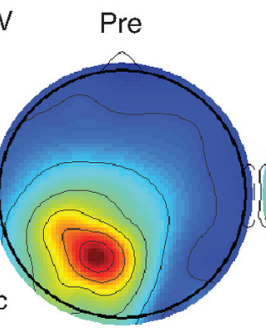

Lower right VF
Figure 2. Topography of the $\mathrm{C} 1$ component of the VEP. Cross-subject average EEG responses to the trained orientation before and after training, for subjects trained in each quadrant are shown at the mean (1 peak time [upper right visual field (VF): $n=3$, upper left VF: $n=4$, lower leftVF: $n=4$, lower rightVF: $n=3$ ]. The posterior focus of maximum amplitude (negative for upper visual field and positive for lower visual field) represents the $\mathrm{C1}$ component, and shows a clear increase in amplitude following training.
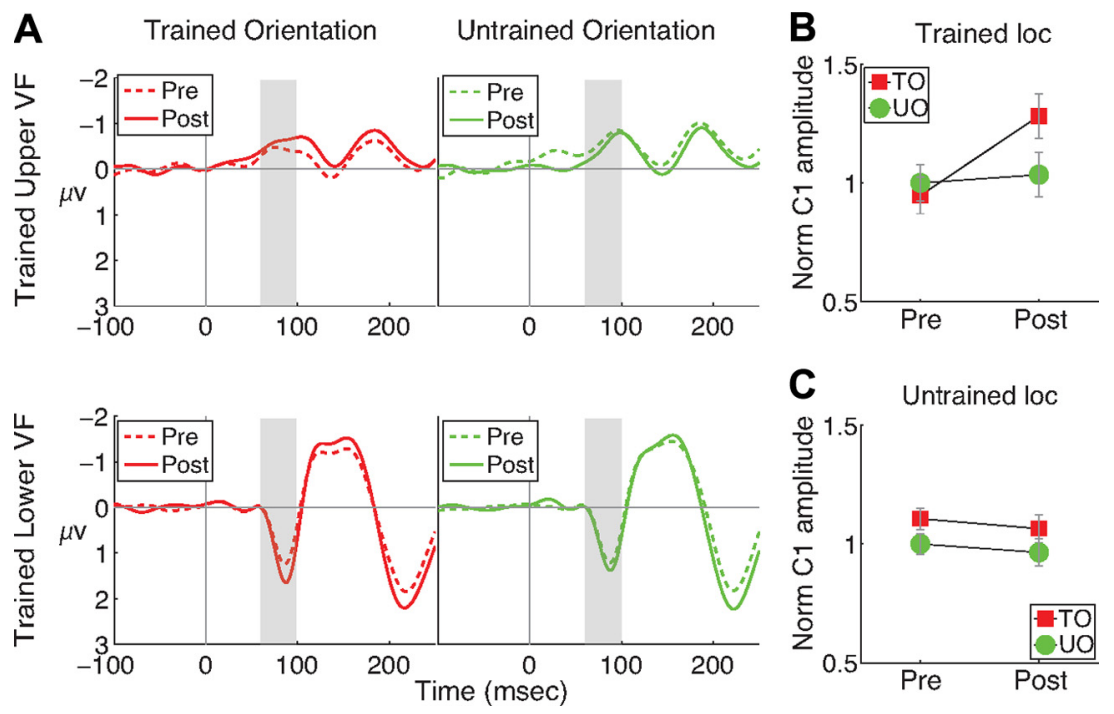

Figure 3. Time course of the VEP. $\boldsymbol{A}$, Grand average VEPs for stimuli presented in the trained quadrant [upper visual field (VF): $n=7$, lower VF: $n=7]$. Subjects trained in the upper and lower visual fields are presented in the upper and lower panels, normalized peak amplitude of the C1 for the trained orientation (TO) and untrained orientation (UO) at the trained (B) and untrained $(\boldsymbol{C})$ locations. Error bars are \pm 1 SEM difference between pretraining and posttraining amplitude.

posttraining). The three factors interacted reliably $\left(F_{(1,13)}=9.10\right.$, $p<0.010)$. Planned comparisons revealed that $\mathrm{C} 1$ amplitude increased more for the trained orientation than for the control orientation only at the trained location $\left(t_{(13)}=3.24, p<0.006\right.$ at the trained location, and $t_{(13)}=0.16, p>0.879$ at untrained locations). The normalized mean amplitude of an early portion of the C1 (70-100 ms after stimulus) (gray bars in Fig. 3A) showed the same pattern of results as did peak amplitude (ANOVA $F_{(1,13)}=$ 9.48, $p<0.009$; planned comparisons $t_{(13)}=2.98, p<0.011$ at the trained location and $t_{(13)}=0.67, p>0.514$ at the untrained locations).

We investigated whether the VEP results showed evidence of learning that was specific for location but generalized across lo- 
cation, by examining whether the untrained orientation showed greater effects of learning at the trained location than at the untrained location (supplemental Fig. S2, available at www. jneurosci.org as supplemental material). A planned comparison showed no significant effect $\left(t_{(13)}=0.70 ; p>0.49\right)$. Thus, our VEP results did not show the location-specific training effect visible in the behavioral data. Spatial attention may have produced this discrepancy. In behavioral testing, the detection task required attention to be focused on the target location, and training likely improved subjects' ability to focus attention at the trained location, resulting in improvements for both orientations. However, during EEG recording, the RSVP task focused attention on the center of the screen. This likely prevented expression of learning due to improved spatial attention.

We also examined the latency of the peak of the $\mathrm{C} 1$ for each stimulus orientation and location. No reliable differences in latency across conditions were found. Our mean latency (92.5 \pm $9.8 \mathrm{~ms}$ ) is within the range found in prior reports, and would have been shorter but for two subjects with relatively long latencies. Removing these two subjects from the analysis did not change the overall pattern of results.

Normalized VEP amplitudes at the trained and untrained locations differed somewhat during pretest: The trained location showed a trend toward an "oblique effect," with smaller amplitudes for the diagonally oriented stimulus than for the horizontally oriented one. The untrained location showed the reverse pattern. Since subjects trained using the diagonal stimulus, we were concerned that the learning effect could depend upon the oblique effect seen at the trained location at pretest. Accordingly, we reran the ANOVA (with orientation, location, and session as factors), while including as a covariate each subject's pretest difference between diagonal and horizontal responses at the trained location (the pretest oblique effect). This analysis still revealed a reliable three-way interaction $\left(F_{(1,12)}=7.75, p<0.017\right)$, our marker of a specific training effect. We also reran the ANOVA including as a covariate the difference between the pretest oblique effects at the trained and untrained location, which also yielded a reliable interaction $\left(F_{(1,12)}=4.69, p<0.051\right)$. ANOVA with covariates (ANCOVA) is the preferred way to compensate for baseline differences in treatment variables such as learning (Overall and Magee, 1992). ANCOVAs on the normalized mean amplitudes (70-100 ms) also found significant three-way interactions $\left(F_{(1,12)}=8.50, p<0.013\right.$, and $\left.F_{(1,12)}=5.85, p<0.032\right)$.

We next examined the relationship between training-induced changes in the $\mathrm{C} 1$ amplitude and behavioral thresholds; no reliable correlation was found ( $p=0.33$ ). Finally, to test whether patterns of eye movements could account for our results, we examined EOG recordings measured during VEP testing for a subset of subjects (supplemental Fig. S3, available at www. jneurosci.org as supplemental material). We tested whether EOG amplitude differed between conditions during the period of the C1 (70-100 ms after stimulus), or immediately before it (50-70 ms after stimulus). EOG amplitudes did not differ reliably between the prelearning and postlearning conditions in either time frame. The EOG traces do suggest the possibility of some small, stimulus-related eye movements, particularly for the vertical EOG for stimuli in the upper visual field, but these cannot account for our results, since amplitudes did not differ as a function of learning.

\section{Discussion}

One month of training on a detection task improved performance by $\sim 30 \%$, and reliably increased the amplitude of $\mathrm{C} 1$, the earliest component of the VEP. The simplest account of these results is that early visual neurons selective for the trained orientation, that provided useful signals for the detection task, increased their gain following training. These neurons then produced greater electrical activity following training, visible in the EEG as increased amplitude of C1.

Two aspects of the $\mathrm{C} 1$ potential led to a consensus that its generator lies in V1. First, its temporal onset makes it the first visible component of the VEP, which would be expected to arise from V1, where the strongest initial afferent volley arrives from the lateral geniculate nucleus. Second, the reversal of polarity for upper versus lower field stimuli is consistent with the anatomy of the calcarine sulcus. The upper visual field is represented on the sulcus's lower bank, while the lower visual field is represented on its upper bank, and because the two banks have surface normals that are often $180^{\circ}$ apart, evoked potentials arising from them would be expected to have opposite polarity (Di Russo et al., 2002). The validity of the polarity reversal as an indicator of a V1 generator has recently been challenged, however (Ales et al., 2010), and so for this paper we will conservatively assume that the C1's generator is in early visual cortex.

A number of results suggest a role for feedback signals, traveling from later to earlier visual areas, in the expression of perceptual learning (for review, see Kourtzi and DiCarlo, 2006). Our results, however, are not easily accounted for by such signals. The simplest feedback model depends upon dynamic visual attention: Subjects might consciously or unconsciously recognize the trained stimulus and increase their attention to it (e.g., Shiffrin and Schneider, 1977), which in turn could amplify signals in early visual cortex. This model cannot explain our results because recognizing the trained pattern and deploying visual attention would take tens of milliseconds, delaying effects beyond the early time periods in which we observed them. A second model of feedback is task-dependent top-down modification of V1 response: When performing a trained task, ongoing feedback from higher areas might reconfigure V1 to amplify relevant information without delay (e.g., Li et al., 2004). This account is also not consistent with our results. During EEG recordings, subjects performed a demanding foveal RSVP letter task, which differed dramatically from the trained eccentric grating detection task.

Some more complex feedback models are also inconsistent with our results. It is theoretically possible, for example, that after learning, a trained stimulus generates very rapid feedback that amplifies V1 response, regardless of task and independent of voluntary attention. Such feedback could be functionally equivalent to feedforward amplification, however, if it arises rapidly whenever the trained stimulus is present, independent of the results of later stages of processing (e.g., task recognition, and allocation of attention). One way in which this model might functionally differ from feedforward amplification could be if the feedback depends upon recognition of the trained stimulus by later visual areas that represent objects. Our results cannot be explained by a model of this sort that depends upon conscious recognition; given the demands of the RSVP task and the rapid presentation of stimuli in all four quadrants, our subjects reported that they were not consciously aware of the orientation of most, if not all, stimuli.

Our results agree with prior work suggesting that perceptual learning can affect responses in early visual cortex (Schoups et al., 2001; Schwartz et al., 2002; Furmanski et al., 2004; Kourtzi et al., 2005; Sigman et al., 2005; Mukai et al., 2007; Yotsumoto et al., 2008). Importantly, our findings do not disagree with results showing that learning can affect neuronal response in later visual areas (Yang and Maunsell, 2004; Raiguel et al., 2006), and areas 
responsible for perceptual and motor decisions (Law and Gold, 2008). Such learning could have occurred in our task; our rapid stimulus design (interstimulus interval of 150-450 ms) may have masked our ability to detect it in the VEP data. Learning likely affects multiple levels of the visual hierarchy, and the relative magnitude of changes in early and late processing stages may depend upon the task (Ahissar and Hochstein, 2004). Detection of the simple patterns may depend relatively directly upon responses in early visual areas (e.g., Hawken and Parker, 1990; Ress and Heeger, 2003), which may have increased the size of early feedforward effects we observed relative to those observed in most other studies.

In the present study, we used low-contrast $(\sim 1 \%)$ gratings for training, but full-contrast gratings in the EEG sessions. Our finding of increased response even for full-contrast gratings suggests that learning can boost response even when neural contrast response functions may have reached an asymptote. This pattern of results corresponds to a "response gain" in which learning scales neural response at all contrast levels, rather than a "contrast gain" in which learning increases the effective contrast of a stimulus. Our data could also result from an increased number of neurons responding to the stimulus.

Our results agree with, and extend, prior studies that have examined perceptual learning using EEG. One line of work established that changes resulting from perceptual learning can be observed in VEP topography and amplitude (Skrandies et al., 2001; Ludwig and Skrandies, 2002; Shoji and Skrandies, 2006). Our study additionally shows that learning can affect the early VEP component, C1. Another study found decreases in the amplitude in the upper visual field $\mathrm{C} 1$ following perceptual learning of a texture segregation task (Pourtois et al., 2008). Our results cover both the upper and lower visual field, and show an increase in $\mathrm{C} 1$ amplitude that is relatively straightforward to interpret. Additionally, by randomizing stimulus order and using a fixation task during testing, our results rule out attention-based and taskbased feedback that was not examined in this previous work.

In summary, the present results suggest that perceptual learning can induce plasticity in early visual cortex through local receptive field changes. Exploring the relative contributions to behavior of these early effects, those originating at higher levels, and those due to feedback from higher to lower levels, remains an open area for future research.

\section{References}

Ahissar M, Hochstein S (2004) The reverse hierarchy theory of visual perceptual learning. Trends Cogn Sci 8:457-464.

Ales JM, Yates JL, Norcia AM (2010) V1 is not uniquely identified by polarity reversals of responses to upper and lower visual field stimuli. Neuroimage 52:1401-1409.

Di Russo F, Martínez A, Sereno MI, Pitzalis S, Hillyard SA (2002) Cortical sources of the early components of the visual evoked potential. Hum Brain Mapp 15:95-111.

Di Russo F, Martínez A, Hillyard SA (2003) Source analysis of event-related cortical activity during visuo-spatial attention. Cereb Cortex 13:486-499.

Fahle M (2004) Perceptual learning: a case for early selection. J Vis $4: 879-890$

Foxe JJ, Simpson GV (2002) Flow of activation from V1 to frontal cortex in humans. A framework for defining "early" visual processing. Exp Brain Res 142:139-150.

Furmanski CS, Schluppeck D, Engel SA (2004) Learning strengthens the response of primary visual cortex to simple patterns. Curr Biol 14:573578 .

Ghose GM, Yang T, Maunsell JH (2002) Physiological correlates of perceptual learning in monkey V1 and V2. J Neurophysiol 87:1867-1888.

Gomez Gonzalez CM, Clark VP, Fan S, Luck SJ, Hillyard SA (1994) Sources of attention-sensitive visual event-related potentials. Brain Topogr 7:41-51.

Hawken M, Parker A (1990) Detection and discrimination mechanisms in the striate cortex of the old-world monkey. In: Vision: coding and efficiency (Blakemore C, ed), pp 103-116. Cambridge, UK: Cambridge UP.

Hua T, Bao P, Huang CB, Wang Z, Xu J, Zhou Y, Lu ZL (2010) Perceptual learning improves contrast sensitivity of V1 neurons in cats. Curr Biol 20:887-894.

Jeffreys DA, Axford JG (1972) Source locations of pattern-specific components of human visual evoked potentials. I. Component of striate cortical origin. Exp Brain Res 16:1-21.

Karni A, Sagi D (1991) Where practice makes perfect in texture discrimination: evidence for primary visual cortex plasticity. Proc Natl Acad Sci U S A 88:4966-4970.

Kelly SP, Gomez-Ramirez M, Foxe JJ (2008) Spatial attention modulates initial afferent activity in human primary visual cortex. Cereb Cortex 18:2629-2636.

Kourtzi Z, DiCarlo JJ (2006) Learning and neural plasticity in visual object recognition. Curr Opin Neurobiol 16:152-158.

Kourtzi Z, Betts LR, Sarkheil P, Welchman AE (2005) Distributed neural plasticity for shape learning in the human visual cortex. PLoS Biol 3:e204.

Law CT, Gold JI (2008) Neural correlates of perceptual learning in a sensory-motor, but not a sensory, cortical area. Nat Neurosci 11:505-513.

Li W, Piëch V, Gilbert CD (2004) Perceptual learning and top-down influences in primary visual cortex. Nat Neurosci 7:651-657.

Lu ZL, Liu J, Dosher BA (2010) Modeling mechanisms of perceptual learning with augmented Hebbian re-weighting. Vision Res 50:375-390.

Ludwig I, Skrandies W (2002) Human perceptual learning in the peripheral visual field: sensory thresholds and neurophysiological correlates. Biol Psychol 59:187-206.

Martínez A, Anllo-Vento L, Sereno MI, Frank LR, Buxton RB, Dubowitz DJ, Wong EC, Hinrichs H, Heinze HJ, Hillyard SA (1999) Involvement of striate and extrastriate visual cortical areas in spatial attention. Nat Neurosci 2:364-369.

Mukai I, Kim D, Fukunaga M, Japee S, Marrett S, Ungerleider LG (2007) Activations in visual and attention-related areas predict and correlate with the degree of perceptual learning. J Neurosci 27:11401-11411.

Overall JE, Magee KN (1992) Baseline correction in a two-way randomized blocks design. J Biopharm Stat 2:205-217.

Poggio T, Fahle M, Edelman S (1992) Fast perceptual learning in visual hyperacuity. Science 256:1018-1021.

Pourtois G, Rauss KS, Vuilleumier P, Schwartz S (2008) Effects of perceptual learning on primary visual cortex activity in humans. Vision Res 48:55-62.

Raiguel S, Vogels R, Mysore SG, Orban GA (2006) Learning to see the difference specifically alters the most informative V4 neurons. J Neurosci 26:6589-6602.

Ress D, Heeger DJ (2003) Neuronal correlates of perception in early visual cortex. Nat Neurosci 6:414-420.

Schoups A, Vogels R, Qian N, Orban G (2001) Practising orientation identification improves orientation coding in V1 neurons. Nature 412:549-553.

Schwartz S, Maquet P, Frith C (2002) Neural correlates of perceptual learning: a functional MRI study of visual texture discrimination. Proc Natl Acad Sci U S A 99:17137-17142.

Shiffrin R, Schneider W (1977) Controlled and automatic human information processing: II. Perceptual learning, automatic attending and a general theory. Psychol Rev 84:127-190.

Shoji H, Skrandies W (2006) ERP topography and human perceptual learning in the peripheral visual field. Int J Psychophysiol 61:179-187.

Sigman M, Pan H, Yang Y, Stern E, Silbersweig D, Gilbert CD (2005) Topdown reorganization of activity in the visual pathway after learning a shape identification task. Neuron 46:823-835.

Skrandies W, Jedynak A, Fahle M (2001) Perceptual learning: psychophysical thresholds and electrical brain topography. Int J Psychophysiol 41:119-129.

Xiao LQ, Zhang JY, Wang R, Klein SA, Levi DM, Yu C (2008) Complete transfer of perceptual learning across retinal locations enabled by double training. Curr Biol 18:1922-1926.

Yang T, Maunsell JH (2004) The effect of perceptual learning on neuronal responses in monkey visual area V4. J Neurosci 24:1617-1626.

Yotsumoto Y, Watanabe T, Sasaki Y (2008) Different dynamics of performance and brain activation in the time course of perceptual learning. Neuron 57:827-833. 